\title{
DE LAS FALSIFICACIONES EN LA HISTORIA: JUAN JOSÉ HEYDECK ( $n$. 1755) Y SU «PORTENTOSO» DESCUBRIMIENTO DE UNA VACUNA CONTRA LA VIRUELA
}

\section{Guillermo Olagüe de Ros}

Universidad de Granada

\section{RESUMEN}

Juan José Heydeck ( $n$. 1755) es una turbia figura que en los últimos años está adquiriendo cierto relieve historiográfico. Se ha sobrevalorado, en exceso, su falso hallazgo de una vacuna contra la viruela en cabras de Madrid. En esta nota, que es respuesta a un artículo aparecido en esta revista en 2006, se revisa historiográficamente a este personaje y se demuestra la falsedad de su hallazgo, fruto de su ambiciosa y desmedida ambición.

PALABRAS CLAVE: España, S. XIX, vacuna, viruela, Juan José Heydeck, falsificaciones en la historia.

ON THE FALSIFICATIONS IN HISTORY: JUAN JOSÉ HEYDECK ( $n$. 1755) AND HIS «AMAZING» DISCOVERY OF A VACCINE AGAINST THE SMALLPOX

\begin{abstract}
Juan Jose Heydeck (b. 1755) is a turbid figure that in the last years he is acquiring certain historical relief. His false find of a vaccine has been overvalued, in excess, against the smallpox in goats of Madrid. In this note, which is a response to an article appeared in Asclepio in 2006, this personage is checked from an historical point of view and there is demonstrated the falsehood of his find, fruit of his excessive ambition.
\end{abstract}

KEY WORDS: Spain, XIXth C., vaccine, smallpox, Juan José Heydeck, falsifications in history.

DEL VALOR DE LA REVISIÓN DE LOS ARTÍCULOS, ANTES DE SU PUBLICACIÓN, PARA DETERMINAR SU CALIDAD CIENTÍFICA

Un indicador que utilizan las revistas científicas para medir la valía de los originales que reciben para su publicación, consiste en someterlos a la previa 
opinión de expertos, lo que en la tradición anglosajona se conoce como peer reviews. A tenor del informe emitido, la revista editará, en ocasiones previo cambios sustanciales, o rechazará definitivamente, el artículo. A pesar de los filtros de exigencia que suelen aplicar las revistas, no siempre se actúa con suficiente rigurosidad. Hace unos años, una prestigiosa revista médica americana, los Annals of Emergency Medicine envió a más de doscientos censores un manuscrito falso que contenía intencionadamente errores importantes y otros menos significativos. Un grupo de los revisores aceptó la edición del trabajo tal como había sido enviado y otro, significativo también, a condición de introducir algunas modificaciones en el original ${ }^{1}$.

Pero a pesar de las lícitas críticas que puedan recibir, es universalmente aceptado que los peer reviews siguen siendo un resguardo de seriedad de cualquier publicación que se precie. Asclepio desde hace años recurre a evaluadores externos para determinar la calidad de los originales que se le remiten de cara su posible publicación. Lo que explica que, gracias a la seriedad de los artículos que finalmente se editan, esta revista se indiza en los más importantes repertorios bibliográficos de ciencias sociales y humanidades.

En el último número publicado hasta la fecha, sin embargo, se incluye un trabajo sobre Juan José Heydeck en el que se le concede un exagerado y desenfocado papel en el curso de la historia de la vacuna jenneriana en España ${ }^{2}$. Como ya he mostrado en otras aportaciones, Heydeck fue un auténtico pícaro que intentó engañar a las autoridades sanitarias y políticas con un pretendido descubrimiento de una vacuna en cabras que, según su argumento, preservaba con la misma eficacia que la vacuna. Esta nota, pues, ofrece una lectura diferente sobre Heydeck y sobre su aventura hispana, que incluso consiguió informes positivos para editar una monografía que era una falsificación en toda regla. Como señalo al fin de esta nota, la adecuada comprensión de un biografiado pasa por una interpretación de las fuentes y no sólo su lectura, una heurística de literatura secundaria lo más completa posible, y la inserción del sujeto de estudio en las corrientes histórico científicas del momento.

1 Baxt, WG.; Waeckerle, JF; Berlin, JA; Callaham, ML (1998). Who review the reviewers?. Feasibility of using a fictitious manuscript to evaluate peer review performance. Ann. Emerg. Med., 33, p. 310-317. Una interesante puesta al día sobre el valor de los peer reviews, y sobre posibles soluciones para paliar fallos significativos en los informes expedidos por los revisores en: CAMPANARIO, J. C. (2002). El sistema de revisión por expertos (peer review): muchos problemas y pocas soluciones. Rev. Esp. Doc. Cient., 25, 166-184.

2 RAmírez Martín, S. M. (2006). Juan José Heydeck, un alemán en la Corte de Carlos IV: experimentos sobre la viruela. Asclepio, 58, p. 165-202. Una primera noticia sobre Heydeck la ofreció en RAmírez MARTíN, S. M.; RAMírez MARTíN, V. (2004). Juan José Heydeck: un humanista en busca de la salud pública. Raíces. Revista Judía del Cultura, 18, (n. 58), p. 58-65. 


\section{LA IMAGEN DE JUAN JOSÉ HEYDECK ( $n$. 1755$)$ EN LA HISTORIOGRAFÍA}

A pesar de que la autora del artículo de Asclepio afirma tajantemente que sobre él «no hay nada escrito» ${ }^{3}$, existe una cierta literatura analítica sobre Juan José Heydeck, en parte panegírica, un apóstata del judaísmo que a fines del siglo XVIII se afincó en España, ejerció la docencia en el madrileño Colegio de San Isidro, y sirvió de intérprete a las autoridades responsables de la Inquisición, entre otras actividades ${ }^{4}$.

\section{JUAN JOSÉ HEYDECK Y LOS TEXTOS EPIGRÁFICOS DE LA SINAGOGA DEL TRÁNSITO DE TOLEDO (1795)}

En 1951 Cecil Roth (1899-1971), profesor de Estudios Judíos en la Universidad de Oxford, experto en el Tribunal de la Inquisición y en la historia de los marranos, publicaba en The Journal of Jewish Studies una nota sobre «Don» Juan José Heydeck . Roth debía ser un buen conocedor de los usos del tratamiento y cortesía en lengua española, pues que entrecomillara Don para referirse a Heydeck era bien significativo. Entre otros epítetos que dedica a Heydeck le tilda de «ignorante converso del judaísmo», «tramposo» y «algo despreciable». En fin, para Roth la vida de Heydeck era un compendio de «confusas y vergonzosas aventuras» que el propio Heydeck construyó para hacer fortuna y granjearse fama y respeto entre sus contemporáneos. Roth, por ejemplo, duda que su padre fuera un rabino de renombre y que el propio Juan José, Moshé Levy o Levy Moshé, antes de su conversión, se formara en Metz y Praga. El hecho es que en 1779 se convirtió al cristianismo como

3 RAMírez MARTín (2006), p. 165.

4 Además de los que consigno en la nota 2, véase también: GARZón, J. I. (2003). Noticias acerca de Juan Joseph Heydeck, apóstata judío en la España del siglo XVIII. Raíces. Revista Judia de Cultura, 17 (n. 54), p. 16-28. El trabajo de Garzón es ponderado y se centra en comentar un texto hebreo de Heydeck, del que reconstruye bastante acertadamente los principales momentos de su vida. Otras menciones a Heydeck se encuentran en los trabajos citados por mí en la nota 28 de mi estudio de 1995, que relaciono en la nota 9 de este artículo, concretamente: Keyserling, M. (1895), Simón DíAZ, J. (1948) y Aguilar Piñal, F. (1968). En dicha nota 28 el lector interesado podrá encontrar las referencias completas de los mismos.

5 Roth, C. (1950-1951). «Don» Juan Josef Heydeck. Journal of Jewish Studies, 2, p. 187-194. He consultado el artículo de Roth muy recientemente, cuando en mi trabajo indicado en la nota 9 (OlAGǗ DE Ros (1995)) ya había advertido de la poca honestidad de Heydeck en su supuesto descubrimiento de la vacuna contra la viruela. 
miembro de la Iglesia de Inglaterra durante su estancia en ese país para, en 1783, bautizarse como católico, volver durante un tiempo otra vez al protestantismo y, finalmente, profesar como convencido católico hasta el final de sus días. Roth cuestiona también que durante su etapa inglesa trabajara en la sección de manuscritos orientales del Museo Británico y que fuera profesor de dichas lenguas en Dublín. Tras un breve paso por los Estados Unidos de América, con fines evangelizadores entre algunas tribus nativas, llegó a España, finalmente, en el verano de 1788.

Con un currículo tan notorio y con fama de respetable rabino converso al catolicismo, hecho que era conocido en España desde cuatro años antes, Heydeck no tuvo excesivos problemas para encontrar un medio de vida en nuestro país, al que llegó con cartas de recomendación traídas de las Américas. En efecto, fue contratado como Profesor de Hebreo en el Estudio de San Isidro de Madrid y se le responsabilizó, también, de los manuscritos y libros orientales de la Biblioteca del Colegio.

A raíz de la trascripción y edición de textos epigráficos hebreos que se conservan en la antigua Sinagoga del Tránsito de Toledo, Heydeck mostró algunas de sus habilidades «intelectuales» que, años después, volvería a esgrimir a raíz del pretendido descubrimiento de linfa vacunal antes citado. En resumen, según Roth, Heydeck no estudió de primera mano dichas inscripciones, si no que se limitó a traducir de nuevo al hebreo las que cinco siglos antes había vertido con no muy buena fortuna al castellano un erudito de la época. Con tan escasa pericia y cuidado que Heydeck incluyó algunos fragmentos que ya habían desaparecido con el paso del tiempo, pero que sí constaban en la obra del renacentista. La Academia de la Historia, a instancias de Godoy, nombró dos expertos que dieron por bueno el trabajo de Heydeck y recomendaron su publicación en 1795, bajo el patronazgo de la Imprenta Real. Pero uno de los Comisionados por la Academia, poco después de dar su placet, descubrió el engaño del alemán, que corroboró una nueva comisión nombrada por la Academia que, incluso, marchó a Toledo para verificar in situ la falacia del judeo-converso ${ }^{7}$. Salvo el episodio que centra esta nota, y

6 El texto en el que se pregona su conversión probablemente se publicó originariamente en alemán, aunque se desconoce copia del mismo, se tradujo al francés en 1783 y al siguiente año se imprimió en castellano en Madrid [RoTH (1950-1951), p. 189].

7 Toda la documentación sobre el enfrentamiento entre Heydeck y la Academia de la Historia, está disponible en la página web de la Biblioteca Virtual Miguel Cervantes [http://www.cervantesvirtual.com]. En la misma se ofrece una reproducción original de varias decenas de documentos manuscritos sobre este enojoso asunto. 
que comentaré a continuación, poco más se sabe de Heydeck. Lo que sí parece claro es que al final de su vida, según Roth, Heydeck se alineó con las fuerzas más reaccionarias y ultramontanas de la España del momento ${ }^{8}$.

JUAN JOSÉ HEYDECK Y SU FALSO DESCUBRIMIENTO DE UNA VACUNA CONTRA LA VIRUELA EN LAS CABRAS DE MADRID (1803-1806)

Si he analizado con cierto detalle las circunstancias de la edición de su obra de 1795 es porque, en relación con el pretendido descubrimiento de linfa contra la viruela en las ubres de cabras de Madrid, Heydeck recurrió, de nuevo, a la mentira, el fraude y la manipulación documental para convencer a los entendidos de su pericia y sagacidad. Pero en esta ocasión el resultado fue infructuoso desde el primer momento, quizás porque su escandalosa participación en la trascripción de los textos de la Sinagoga toledana, debía de ser ya bien conocida en los círculos intelectuales del Madrid de entonces como para concederle el mínimo crédito en esta nueva aventura. Por este motivo buscó apoyos a su ficticio descubrimiento fuera de España, tergiversando el sentido de algunos documentos oficiales en beneficio propio. Con cierto detalle analicé este episodio en una publicación anterior sobre la introducción de la vacuna contra la viruela en España. Me limitaré en esta nota, pues, a resumir los puntos más notorios del mismo9.

En febrero de 1803, cuando los ecos del escándalo de la Sinagoga estaban amortiguados, Heydeck comunicó por carta al ministro Pedro Cevallos que había descubierto en las ubres de unas cabras de un pastor madrileño vecino del estudio de San Isidro unas pústulas cuyo fluido, según su opinión, preservaba contra la viruela con la misma eficacia que el de las vacas inglesas. En unos momentos en que el desabastecimiento de pus efectivo podía ser un problema, la noticia era francamente buena, pues iba a permitir que nuestro país ya no tuviera que depender en el futuro de la traída de fluido contra la viruela

8 Roth (1950-1951), p. 193-194.

9 OlagüE DE Ros, G. (1995), La introducción de la vacunación jenneriana en España (1799-1805). En: BaronA, J.L. (ed.). Malaltia i Cultura. Trobades. Seminari d'Estudis sobre la Ciència. Valencia, Seminari d'Estudis sobre la Ciència, p. 251-273 (p. 267-271). Este episodio también lo he recordado en otras publicaciones posteriores, la más reciente, precisamente, en esta misma revista hace un par de años (OlaGÜE DE ROS, G. ASTRAin GALlart, M. (2004) ¡Salvad a los niños!: los primeros pasos de la vacunación antivariólica en España (1799-1805). Asclepio, 56, 7-31). 
desde el extranjero. En marzo de ese año, el Protomedicato encargaba a Pedro Hernández e Ignacio María Ruiz de Luzuriaga, académicos de la Nacional de Medicina, realizar las oportunas pruebas para verificar el aserto del alemán. La pretendida vacunación de una hija de Heydeck no pudo confirmarse que fuera debida al pus caprino, pues lo había sido también con fluido vacuno auténtico, por lo que era difícil dirimir a cuál de las dos se debía el aparente éxito de la misma. Las célebres cabras de Heydeck, según su pastor, tenían fogaradas o lobanillos, opinión que compartía el propio Ruiz de Luzuriaga, y la vacunación que se efectuó en una hija de Díaz Canedo, cirujano y yerno de Pedro Hernández, no dio ningún fruto positivo, a igual que una posterior -el 26 de mayo - también efectuada por Díaz Canedo a dos criaturas, episodio sobre el que luego volveré. De una planificada vacunación masiva a los niños de la casa de Desamparados madrileña con el fluido caprino, que debían supervisar Ruiz de Luzuriaga y Hernández, no se conserva noticia alguna ${ }^{10}$, aunque en nota reservada dirigida a la Academia de Medicina, el primero descalificaba totalmente el invento de Heydeck y lo consideraba una quimera.

Dos años después, Heydeck volvió a pregonar la bondad y plena eficacia de su descubrimiento. En vistas del poco éxito de su anterior aventura, buscó en el extranjero los avales necesarios para definitivamente convencer a las autoridades médicas y políticas españolas de su hallazgo. En plena efervescencia de su descubrimiento, William Andrew, un médico inglés de paso por Madrid, publicó en 1803 en la revista Medical and Physical Journal una nota dirigida a Richard Dunning, notable miembro del Instituto de Vacunación londinense que presidía Jenner, en la que reconstruía la historia del hallazgo del alemán desde febrero hasta mayo de ese año. Los textos del trabajo de Andrew obviamente estaban en inglés, y no me cabe duda alguna de que fuera el propio Heydeck quien se los tradujo del castellano a esa lengua ${ }^{11}$. Además de la comunicación a Pedro Cevallos de su descubrimiento, la respuesta de éste, la Real Orden por la que se invitaba al Real Colegio de Médicos madrileño a realizar los experimentos, y el nombramiento de los comisionados por la Academia de Medicina de Madrid, se ofrecía el informe de los mismos sobre los resultados de la vacunación con el pus de la cabra en los dos niños,

10 En esta primera tentativa parece ser que fueron vacunados cuarenta niños, pero no se sabe el resultado (ANDREW (1803), op. cit. en nota siguiente, p. 345).

11 Andrew, W. (1803). To Mr. Dunning, Plymouth Dock. Med. Phys. J., n. 56, p. 339345, Andrew, en un breve texto introductorio, ya advierte que durante su permanencia en Madrid trabó una estrecha relación con Heydeck (p. 339). Una copia de este artículo se encuentra en el Archivo Histórico Nacional de Madrid, Estado, Leg. 2932 (2), doc. 5. 
Nicolás y María. El informe de Díaz Canedo, que fue el encargado de practicar las vacunas, y cuyo nombre no aparecía como firmante del documento en la nota inglesa, difería sustancialmente del que se ofrecía en la revista británica, según la cual era obra de los comisionados por «The Royal College of Physicians». Para Díaz Canedo la cabra «tenía una erupción en las tetas y cerca de sus pezones parecida a las viruelas naturales, cuando están en su perfecta supuración, con la diferencia que dichos granos carbuncos tenían muy poca areola y son más chatos o aplanados que las viruelas naturales...»; concluía Díaz Canedo que el pus, que conservó en cristales, «se ve en todo diferente al fluido vacuno». En el informe que recogía la revista inglesa la cabra presentaba «pock», y sus pústulas «were full of virulent matter and fit to extract for inoculation».

El relato sobre el curso temporal de la vacunación con la linfa de la cabra en los niños inserto en la revista londinense traslucía claramente que la experiencia había sido un éxito, pero en el documento oficial de Díaz Canedo se ponía en evidencia, de manera taxativa, que el pus hubiera producido ningún efecto preservativo contra la viruela en las dos criaturas ${ }^{12}$.

La cuestión es que, como resultas de este episodio, Edward Jenner y Dunning dirigieron una carta a Heydeck en 1806 felicitándole por tan buena noticia. Inmediatamente Heydeck se cuidó de hacérsela llegar al Ministro Cevallos que, presionado por la autoridad y prestigio de los firmantes, propuso hacer una vacunación masiva en los niños de la Inclusa madrileña. La oposición de la Junta de Señoras de la misma, por el precario estado físico de los niños, impidió afortunadamente cumplir con el deseo. Aprovechando la carta de los ingleses, Heydeck solicitaba al Ministro una gratificación económica como recompensa por su notable «invento». No era la primera vez. Por cinco veces, y con ningún éxito, Heydeck había solicitado remuneraciones económicas para subvenir a las necesidades de su numerosa familia ${ }^{13}$

Este episodio, más propio de un relato de la picaresca española que de un hallazgo científico, no fue el único en estos años en relación con la vacuna contra la viruela. Otros dos pícaros, Rafael Costa de Quintana en 1803, y Marcelo Hortet y Pauló, entre 1804 y1806, de los que ya di cuenta hace tiempo, intentaron también obtener ganancias económicas y cierto prestigio, con el falso descubrimiento de linfa contra la viruela en otras especies autóctonas de la cabaña ganadera nacional. Costa de Quintana, por ejemplo, sabedor de la proyectada expedición de Balmis, puso todo su empeño en convencer a las

12 OlagüE DE Ros (1995), p. 270.

13 GARZÓN (2003), p. 21 
autoridades de que en Mondoñedo había vacas con igual enfermedad que las inglesas, por lo que sugirió, además de recibir él mismo una pensión por tan feliz hallazgo, transportar las vacas leonesas en el navío expedicionario ${ }^{14}$.

\section{DEL VALOR DE LA BIOGRAFÍA COMO GÉNERO HISTORIOGRÁFICO}

En julio de 2004 la Sociedad Española de Historia de la Medicina celebró su XIII Simposio en Jaca, que dedicó monográficamente a estudiar la validez de la biografía como género historiográfico. Los resultados de la reunión se publicaron en esta misma revista en el primer número del año 2005. En mi aportación expuse que la reconstrucción de la vida de los médicos del pasado es un modelo de hacer historia perfectamente válido e interesante, y que contribuye de forma positiva a una mejor comprensión del pasado ${ }^{15}$.

Uno de los problemas más frecuentes a la hora de confeccionar una biografía se da cuando el estudioso acaba por santificar al sujeto de su estudio, conclusión a la que se suele llegar cuando se da una identificación absoluta con el sujeto estudiado, es decir, cuando se tiene una visión excesivamente positivista y beatífica de la ciencia y de los científicos. Si un individuo hace ciencia, y la ciencia es intrínsecamente buena, el que la produce es, por tanto, honesto y bueno. Además de esta razón, que es importante, hay otras que desvirtúan la objetividad a la hora de confeccionar una biografía. No cabe duda que el ansia de novedad por el historiador es razón de peso; pero en muchos casos esa aparente originalidad suele ser resultado de una deficiente heurística previa que convierte en novedoso lo ya sabido. Otra causa es no apreciar que en la vida de un personaje hay varias biografías y que todas ellas convergen en el momento de su máxima plenitud. La cabal comprensión de todos y cada uno de los episodios biográficos de un personaje permite, no cabe duda, tener una visión más completa del mismo. Finalmente, cierto precursorismo de tinte localista, es decir, la búsqueda forzosa de una mente prodigiosa que, después del descubridor, aplicó o mejoró el hallazgo de aquél en un escenario geográfico y lingüístico diferente.

Los datos con los que un científico confecciona una doctrina no son ciencia ni conocimiento. Adquieren sentido cuando, precisamente, se integran en

14 Acerca de Hortet y Pauló: OlagüE De Ros (1995), p. 271-273; Sobre Costa de Quintana: OlagüE De Ros; Astrain Gallart (2004).

15 OlagüE De Ros, G. (2005). De las vidas ejemplares a las biografías colectivas de los médicos. Una perspectiva crítica. Asclepio, 57, p. 135-148. 
una hipótesis explicativa de una parcela de la realidad, que se comunica, es decir, recibe una sanción social por la comunidad de científicos. Por tanto, el creador de cualquier teoría debe convencer o persuadir a los demás de la autenticidad de la misma conforme a los supuestos que son propios de la ciencia, es decir, la demostración validada. En la historia de Heydeck se dan una serie circunstancias especiales. Los actores que intervinieron fueron, por un lado, los médicos y cirujanos, que desde el comienzo se mostraron sumamente cautos ante el descubrimiento, y por otro el propio Heydeck, sin cualificación médica y con un pasado próximo algo singular, que ante una observación casual, como fue también la Jenner, quiso elevarla al rango de hallazgo trascendente. Las reticencias de los expertos, que en sus demostraciones invalidaban las tesis del alemán y, por tanto, suponían una barrera a sus aspiraciones, las quiso disipar recurriendo a un criterio de autoridad, la de los médicos ingleses y, de paso, obtener unos beneficios económicos que, en su opinión, le correspondían plenamente. Pero, al igual que durante su aventura con los textos sinagogales de Toledo, recurrió al engaño y a la mentira. La falsificación, obviamente, no es de recibo, y es tarea del historiador, cuando la advierte, publicitarla y denunciarla.

Fecha de recepción: 15 de enero de 2007

Fecha de aceptación: 2 de febrero de 2007 
